\title{
100 ans après la prédiction d'Einstein, la SFP salue la détection interférométrique des ondes gravitationnelles
}

Le 11 février 2016 restera une date importante dans I'histoire des sciences, avec la publication dans la revue Physical Review Letters de la première détection d'ondes gravitationnelles induites par la coalescence de deux trous noirs lointains très massifs $(*)$.

La méthode est fondée sur l'infime déplacement des miroirs d'interféromètres optiques provoqué par le passage rapide d'une onde de gravitation qui distord l'espace-temps, à la suite d'un événement particulièrement violent dans le cosmos. La performance expérimentale est extraordinaire : pour pouvoir distinguer du bruit un aussi petit phénomène, il est nécessaire que plusieurs interféromètres optiques le détectent en même temps en divers points éloignés les uns des autres sur la Terre. C'est ainsi que le signal révélé au public (fig. 1) a été enregistré le 14 septembre 2015 en coïncidence par les deux interféromètres LIGO situés aux États-Unis, dans le cadre de la collaboration LIGO-VIRGO.

Il s'agit d'un travail collaboratif de plus de vingt ans, rassemblant 136 équipes et institutions d'une vingtaine de pays à travers le monde. De nombreux laboratoires français y ont participé à travers VIRGO, le grand interféromètre laser franco-italien situé à Pise, né des idées visionnaires d'Adalberto Giazotto et Alain Brillet (ARTEMIS-CNRS). Ainsi, le Laboratoire des Matériaux Avancés de Villeurbanne a fourni les miroirs à la fois pour VIRGO et pour LIGO. Ces miroirs sont exceptionnellement performants en matière de réflectivité : seule une part par million de la lumière est perdue à chaque réflexion. Le contrôle du bruit et la stabilité des lasers YAG

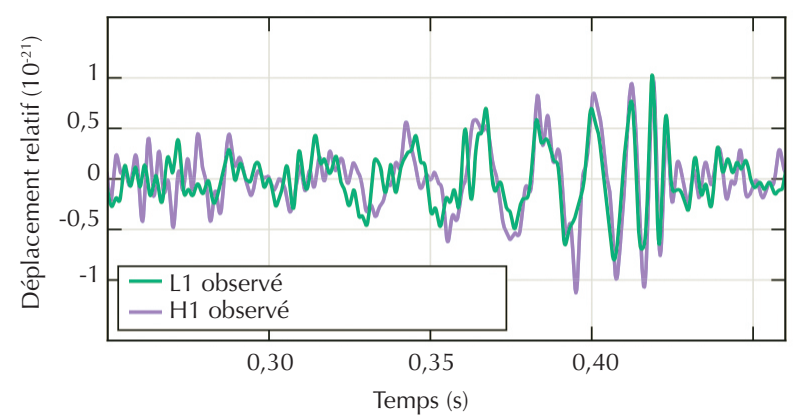

1. L'événement observé par les détecteurs de l'expérience LIG0 (États-Unis) le 14 septembre 2015, à 11 h 51 (heure de Paris). Signaux en mauve (détecteur de Hanford H1) et en vert (détecteur de Livingston L1), indiquant le passage d'une onde gravitationnelle détectée en coïncidence par les deux interféromètres optiques géants de LIG0. En ordonnée, le déplacement relatif des deux miroirs de l'interféromètre à plusieurs kilomètres l'un de l'autre. Les franges d'interférence de l'instrument bougent d'une extrêmement petite fraction de la largeur d'une frange ( $10^{-21}$ en valeur relative). à 1,6 $6 \mathrm{~m}$ utilisés dans les interféromètres représentent également une performance sans précédent de l'optique. L'école française de la gravitation relativiste, impulsée par Thibault Damour (IHES) pour décrire de manière analytique les phénomènes de coalescence, a été elle aussi fondamentale.

Tout comme l'Académie des sciences, la Société Française de Physique salue la naissance d'une nouvelle astronomie : "Une nouvelle fenêtre s'ouvre sur l'Univers. Un siècle après leur prédiction par Einstein, les ondes gravitationnelles en provenance de l'Univers lointain viennent d'être détectées sur Terre... »**).

De nouveaux résultats de la collaboration LIGO-VIRGO sont attendus prochainement. L'interféromètre VIRGO, non utilisé lors de l'enregistrement du premier signal, est maintenant pleinement opérationnel. Son fonctionnement en coïncidence avec les deux instruments de LIGO, va permettre de localiser les événements sources avec un incomparablement meilleur positionnement dans l'espace, par la classique méthode de triangulation utilisée dans le fonctionnement du GPS.

Les moissons scientifiques ont été exceptionnellement fructueuses ces trois dernières années pour la compréhension des lois fondamentales de l'Univers. Cette avancée majeure apporte la pièce manquante dans l'édifice de la théorie de la gravitation d'Einstein. Avec les autres découvertes récentes, le boson de Higgs et les observations du fond cosmologique par le satellite Planck, l'humanité atteint un nouveau palier de la connaissance dans la compréhension du monde physique et de ses lois fondamentales, de l'échelle quantique à l'échelle de l'Univers. De ce socle scientifique solide mêlant théorie, expérience et technologie, naitront, à n'en pas douter, de nouvelles interrogations fascinantes, car telle est la quête essentielle de la science et des scientifiques.

Michel Spiro, président de la SFP Michèle Leduc et Olivier Dulieu, coordinateurs de ce numéro

(*) B.P. Abbott et al., Phys. Rev. Lett. 116 (2016) 061102.

$\left(^{* *}\right)$ Lettre électronique d'information de l'Académie des sciences, numéro 120 : "L'Académie des sciences salue la naissance d'une nouvelle astronomie ".

- Pour revoir la conférence de presse du CNRS, le 11 février 2016 http://webcast.in2p3.fr/videospoint_sur_les_recherches_dondes_ gravitationnelles_par_les_collaborations_virgo_et_ligo 